\title{
Fertilitätserhalt bei Patientinnen mit Mammakarzinom - eine aktuelle Übersicht
}

\section{Fertility preservation in female patients with breast cancer - a current overview}

Autoren

Veronika Guenther ${ }^{1}$, Ibrahim Alkatout ${ }^{1}$, Wiebe Junkers², Dirk Bauerschlag ${ }^{1}$, Nicolai Maass ${ }^{1}$, Soere von Otte ${ }^{2}$

Institute

1 Klinik für Gynäkologie und Geburtshilfe, UKSH Campus Kiel, Kiel

2 Universitäres Kinderwunschzentrum, MVZ, Universitätsklinikum Schleswig-Holstein, Campus Kiel, Kiel

Schlüsselwörter

Mammakarzinom, Fertilitätserhalt, Kryokonservierung, Oozyten, Ovargewebe, GnRH-Agonisten

Key words

breast cancer, fertility preservation, cryopreservation, oocytes, ovarian tissue, GnRH agonist

Bibliografie

DOI https://doi.org/10.1055/a-0578-1019

Senologie 2018; 15: 41-47

(c) Georg Thieme Verlag KG, Stuttgart · New York

ISSN 1611-6453

Korrespondenzadresse

Dr. Veronika Guenther MD

Klinik für Gynäkologie und Geburtshilfe, UKSH Campus Kiel, Arnold-Heller-Straße 3 (Haus 24), 24105 Kiel, Deutschland

Veronika.Guenther@uksh.de

\section{ZUSAMMENFASSUNG}

Bei vielen prämenopausalen Patientinnen, die an einem Mammakarzinom erkranken, ist die Familienplanung noch nicht abgeschlossen, sodass für den Erhalt des fertilen Potenzials Maßnahmen der Fertilitätsprotektion sinnvoll sind. Durch eine Polychemotherapie - unabhängig ob im neoadjuvanten oder adjuvanten Setting - kommt es zu einer irreversiblen Schädigung der Follikel, was unter Umständen zu einer permanenten Infertilität führen kann. Abhängig von den verwendeten Zytostatika und der altersabhängigen Ovarialreserve der Frau, muss das gonadotoxische Risiko als niedrig, mittel oder hoch eingeschätzt werden. Möglichkeiten des Fertilitäts- erhalts sind: a) die Kryokonservierung von fertilisierten oder unfertilisierten Oozyten. Hierbei werden nach ovarieller Hyperstimulation reife Oozyten mittels transvaginaler Follikelaspiration gewonnen und im Anschluss entweder unfertilisiert oder nach erfolgter IVF- oder ICSI-Behandlung kryokonserviert. Bei b) der Kryokonservierung von Ovarialgewebe wird mithilfe eines laparoskopischen Eingriffs etwa $50 \%$ des Ovarkortex eines Ovars reseziert und kryokonserviert. Die Verwendung von c) GnRH-Agonisten als medikamentöse Therapieoption unternimmt den Versuch einer endokrinen Ovarialsuppression, um Oozyten, Granulosa- und Thekazellen vor dem zytotoxischen Einfluss der Chemotherapie zu schützen.

\section{ABSTRACT}

Many premenopausal patients who develop breast cancer have not yet completed their family planning, so measures of fertility protection to preserve their fertile potential would be beneficial. Polychemotherapy causes irreversible damage to the ovarian follicles - irrespective of whether in a neoadjuvant or adjuvant setting - and this can sometimes result in permanent infertility. Depending on which cytostatic agents are used and on the age-related ovarian reserve of the woman, gonadotoxic risk must be classified as low, moderate or high. Options of fertility preservation include: a) cryopreservation of fertilised or unfertilised oocytes. After ovarian hyperstimulation, mature oocytes are retrieved by transvaginal follicle aspiration, after which they are cryopreserved, either unfertilised or on completion of IVF or ICSI treatment. During b) cryopreservation of ovarian tissue, about $50 \%$ of the ovarian cortex of one ovary is resected with the aid of a laparoscopic procedure and cryopreserved. The application of c) $\mathrm{GnRH}$ agonists as a medicinal therapy option is an attempt at endocrine ovarian suppression in order to protect oocytes, granulosa cells and theca cells from the cytotoxic effect of chemotherapy. 


\section{Einleitung}

Die Überlebensrate von Karzinompatientinnen ist in den vergangenen Jahrzehnten dank stetiger Optimierung und Effizienz onkologischer Therapiekonzepte kontinuierlich gestiegen [1].

Krebserkrankungen stellen überwiegend eine Erkrankung des älteren Menschen dar, während hingegen Heranwachsende und junge Erwachsene relativ selten davon betroffen sind. In Deutschland erkranken derzeit pro Jahr etwa 15000 Patienten im Alter zwischen 15 und 39 Jahren an einem Karzinom, bei 480000 Neuerkrankungen insgesamt [2]. In dieser Altersspanne der unter 40-Jährigen ist das Mammakarzinom mit ca. 2500 Erkrankungen pro Jahr in Deutschland die häufigste Krebserkrankung [2, 3]. In der Altersgruppe der unter 45-Jährigen lag die Inzidenz in Deutschland 2011 - 2012 bei 120/100 000 [4]. Viele dieser Frauen haben ihre Familienplanung noch nicht abgeschlossen, was unter anderem auf das steigende Alter der Frauen bei der Geburt des 1. Kindes zurückzuführen ist [6].

Die Tumorbiologie bei jungen Mammakarzinompatientinnen zeigt häufig ein aggressives Wachstumsverhalten und ist mit einer schlechten Prognose assoziiert [7]. Daher stellt die Polychemotherapie gerade bei jungen Patientinnen mit einem Mammakarzinom - meist im neoadjuvanten Setting - eine wichtige Therapiemaßnahme dar.

Die Langzeitfolgen der onkologischen Therapien rücken, insbesondere vor dem Hintergrund der immer späteren Realisierung des Kinderwunsches, in den Fokus. Die prämature Ovarialinsuffizienz („premature ovarian failure“ - POF) ist eine häufige Konsequenz einer Chemotherapiebehandlung. Die Zytotoxizität ist bei Organen mit hoher Zellteilungsrate, wie z. B. Knochenmark, Gl-Trakt oder Thymus, reversibel. Die Ovarien hingegen werden aufgrund ihrer begrenzten Zellzahl mit fehlender Replikation und Regeneration der Follikel durch die Chemotherapie in unterschiedlichem Ausmaß geschädigt [8, 9]. Dies kann zu einer permanenten Infertilität der betroffenen Frauen führen, siehe - Tab. 1, 2.

\section{Premature Ovarian Failure}

Die Auswirkung einer zytotoxischen Therapie auf die Ovarien wird u. a. durch die Störung der steroidproduzierenden Granulosa- und Thekazellen erklärt, in deren Folge die durch sie umschlossenen Oozyten zugrunde gehen [10]. Histologisches Korrelat ist ein Verlust von Follikelstrukturen in den Ovarien mit Fibrosierungen [11].

Das Ausmaß der gonadotoxischen Wirkung einer Chemotherapie hängt erheblich von der verwendeten Substanz und der kumulativen Dosis ab. Besonders Anthrazykline oder alkylierende Substanzen wie Cyclophosphamid zeichnen sich durch eine hohe Zytotoxizität aus, da sie im Gegensatz zu anderen Zytostatika keine Zellzyklus-Spezifität aufweisen und besonders auf nicht proliferierende Zellen zytotoxisch wirken [12]. Basierend auf ihrem Gonadotoxizitätsrisiko kann man die Chemotherapeutika in verschiedene Risikogruppen einteilen ( $\triangleright$ Tab. 1) [13]. Angegeben ist jeweils das Risiko für eine fortbestehende Amenorrhö nach abgeschlossener Chemotherapie. \ Tab. 2 zeigt Amenorrhöraten nach Chemotherapie am Beispiel von ausgewählten Studien [14 - 16].
- Tab. 1 Amenorrhöraten nach Chemotherapie bei prämenopausalen Patientinnen mit Mammakarzinom, Übersicht, nach [13].

\begin{tabular}{|c|c|c|}
\hline $\begin{array}{l}\text { Alter } \\
\text { (Jahre) }\end{array}$ & Chemotherapie & Amenorrhörate (\%) \\
\hline$>40$ & $6 \times \mathrm{CMF}, 6 \times \mathrm{FEC}, 6 \times \mathrm{FAC}$ & > 80 (hohes Risiko) \\
\hline$<40$ & EC (dosisdicht) & \\
\hline $30-39$ & $6 \times \mathrm{CMF}, 6 \times \mathrm{FEC}, 6 \times \mathrm{FAC}$ & 20 - 80 (mittleres Risiko) \\
\hline$>40$ & $4 \times A C$ & \\
\hline$<30$ & $6 \times \mathrm{CMF}, 6 \times \mathrm{FEC}, 6 \times \mathrm{FAC}$ & <20 (niedriges Risiko) \\
\hline$<40$ & $4 \times A C$ & \\
\hline
\end{tabular}

Cyclophosphamid ist am besten untersucht und wird am häufigsten in Zusammenhang mit einer Gonadotoxizität diskutiert: Es besteht ein 4-fach höheres Risiko für die Entwicklung einer prämaturen Ovarialinsuffizienz im Vergleich zu anderen Chemotherapeutika [17]. Des Weiteren spielt das Alter der Patientin für die Entwicklung und Dauer der Ovarialinsuffizienz eine wichtige Rolle: Ältere Frauen, die bereits eine physiologische Reduktion ihres Primordialfollikelpools aufweisen, haben ein höheres Risiko für die Entwicklung einer Infertilität; zudem bewirkt Cyclophosphamid bereits in niedrigeren Dosen eine Gonadotoxizität, als es bei jüngeren Frauen der Fall wäre [18]. Weitere Einflussfaktoren auf die chemotherapieinduzierte Infertilität sind [19]:

- die individuelle ovarielle Reserve und Pharmakokinetik bez. der Metabolisierung,

- die Gesamtdosis,

- die Dosisdichte,

- die Dauer der Behandlung.

Eine Reihe von Studien hat sich mit der Etablierung möglicher Biomarker zur Prädiktion einer chemotherapieassoziierten Amenorrhö bei Mammakarzinompatientinnen beschäftigt [20 - 22]. Ruddy et al. [20] konnten anhand einer multizentrischen, randomisierten Studie mit 124 prämenopausalen Patientinnen zeigen, dass das vor der Chemotherapie gemessene Anti-Müller-Hormon (AMH) als Biomarker für eine chemotherapieassoziierte Amenorrhö fungieren kann. 12 Monate nach abgeschlossener Chemotherapie hatten $82 \%$ der Patientinnen eine Amenorrhö, wobei hier lediglich das höhere Alter als signifikanter Marker zu werten war $(p=0,0003)$. 18 Monate nach Chemotherapie und bei immer noch $81 \%$ iger Amenorrhörate waren sowohl höheres Alter (OR 1,18, 95\%-KI 1,04-1,34, p=0,008) als auch ein vor der Chemotherapie niedriger AMH-Spiegel (OR 0,41, $95 \%-K I$ 0,18-0,95, $p=0,04$ ) ein signifikanter Prädiktor für eine Amenorrhö [20]. Eine Erhöhung des AMH-Wertes vor der Chemotherapie um $1 \mathrm{ng} / \mathrm{ml}$ würde eine Risikoreduktion um 59\% für die Entwicklung einer 18-monatigen Amenorrhö bedeuten. Eine Erhöhung des Alters um ein Jahr hätte eine $18 \%$ höhere Wahrscheinlichkeit für eine 18-monatige Amenorrhö zur Folge [20]. 
> Tab.2 Amenorrhöraten nach Chemotherapie bei prämenopausalen Patientinnen mit Mammakarzinom, ausgewählte Studien [14 - 16].

\begin{tabular}{|l|l|l|l|l|}
\hline Chemotherapie & Alter (Jahre) & Anzahl Patientinnen & Amenorrhörate (\%) & Studie \\
\hline EC/Pac & mittleres Alter: 42 & $\mathrm{n}=80$ & 46,6 & Zhou, 2012 \\
\hline EC/Doc & $<35$ & $\mathrm{n}=166$ & 15 & Fornier, 2005 \\
\hline TAC & prämenopausal & $\mathrm{n}=109$ & 57,7 & Martin, 2005 \\
\hline FAC & prämenopausal & $\mathrm{n}=409$ & 52 & Martin, 2005 \\
\hline $\begin{array}{l}\text { EC= Epirubicin, Cyclophosphamid; Pac = Paclitaxel; Doc = Docetaxel; TAC= Docetaxel, Doxorubicin, Cyclophosphamid; FAC = 5-Fluoruracil, Doxorubicin, } \\
\text { Cyclophosphamid. }\end{array}$
\end{tabular}

Tamoxifen, als endokrine Therapie für eine Dauer von mindestens 5 Jahren bei hormonrezeptorpositivem Mammakarzinom, ist mit keiner Beeinträchtigung der ovariellen Funktion assoziiert [19]. Dennoch muss von einer altersbedingten Minderung der Fertilität durch die Dauer der antiendokrin ausgerichteten Tamoxifeneinnahme ausgegangen werden. Eine Schwangerschaft sollte, alleine schon wegen teratogener Eigenschaften von Tamoxifen, während dieser Zeit vermieden werden [23]. Am Ende der antiendokrinen Therapie ist ein Großteil der Patientinnen peribis postmenopausal. Aus diesem Grund kann mit der Patientin ein Pausieren der Tamoxifentherapie nach einem etwa 2-jährigen Einnahmeintervall diskutiert werden, um zunächst den Kinderwunsch zu realisieren und nachfolgend die Tamoxifeneinnahme zu komplettieren. Dieser innovative Ansatz ist zurzeit Gegenstand wissenschaftlicher Überprüfungen [24, 25].

Der potenzielle Fertilitätsverlust bedingt durch eine Chemotherapie führt zu einer verheerenden psychischen Belastung bei vielen Patientinnen [26], häufig begleitet von depressiven Episoden, Angst, Trauer, Wut, Zerbrechen von Beziehungen, sowie einer verminderten Lebensqualität im Allgemeinen [27, 28]. Entsprechend wird dem Erhalt der ovariellen Funktion während und nach der Chemotherapie, sowohl in Hinblick auf die Fertilität als auch auf den Erhalt der Knochen- und kardiovaskulären Gesundheit, eine besondere Bedeutung beigemessen.

\section{Möglichkeiten des Fertilitätserhalts}

Im Mai 2006 wurde das Netzwerk FertiPROTEKT in Heidelberg von einer Gruppe aus 40 universitären Reproduktionsmedizinern gegründet. Nach über 10 Jahren hat das Netzwerk heute den Status eines eingetragenen Vereins (e. V.) und ist erheblich gewachsen. Reproduktionsmediziner und Biologen von mittlerweile über 100 universitären sowie nicht universitären Zentren in Deutschland, Österreich und der Schweiz haben sich zusammengeschlossen, um flächendeckend fertilitätserhaltende Maßnahmen bei Chemound Strahlentherapie nach einheitlichen Empfehlungen anzubieten [29].

\section{Kryokonservierung von unfertilisierten und fertilisierten Oozyten}

Wenn vor einer geplanten Chemotherapie noch ausreichend Zeit für eine ovarielle Hyperstimulation vorhanden ist, können reife Oozyten durch transvaginale Follikelaspiration gewonnen werden. Diese können durch IVF- oder ICSI-Behandlung fertilisiert und im Pronukleus-(2-Vorkern-)Stadium kryokonserviert werden. In Deutschland ist nur die Kryokonservierung fertilisierter Oozyten rechtlich zulässig, wohingegen in anderen Ländern auch Embryonen konserviert werden können.

Eine Stimulation kann unabhängig vom Zyklustag der Patientin jederzeit begonnen werden („random start“) und dauert etwa 14 Tage [30]. Bewährt hat sich ein Stimulationsprotokoll unter Verwendung eines Aromataseinhibitors (AI) zeitgleich zur Gonadotropingabe [31]. Dieser führt zu einer Senkung des Östradiolspiegels im Plasma bei gleichzeitiger Induktion der Östrogenbildung aus Androgenen in den Granulosazellen. Somit können beide Effekte des Al optimal genutzt werden. Auf der einen Seite werden die messbaren Östradiol-(E2-)Spiegel im Blut maximal supprimiert, auf der anderen Seite wird die Ovulationsinduktion unterstützt [32]. Oktay et al. konnten zeigen, dass eine Stimulation im Antagonistenprotokoll mit FSH kombiniert mit Letrozol (Al der 3. Generation) im Vergleich zu einer Stimulation im Agonisten-Langprotokoll zu einer signifikanten Reduktion der E2-Spiegel $(p<0,001)$ bei gleichbleibender Anzahl an gewonnenen Oozyten $(p=0,43)$ führt [32]. Allerdings muss darauf hingewiesen werden, dass die Stimulation mit Letrozol nicht pauschal, sondern lediglich bei hormonrezeptorpositiven Tumoren zu empfehlen ist. Zudem müssen die Patientinnen über den Off-Label-Use dieses Stimulationsprotokolls aufgeklärt werden.

Wenn die Patientin keinen Partner hat, werden die Oozyten im unfertilisierten Zustand kryokonserviert. Dieses Verfahren sollte aufgrund der hohen Trennungs- und Scheidungsraten grundsätzlich jeder Frau - egal ob ledig, in einer festen Beziehung oder verheiratet - angeboten werden. Hierbei muss gewährleistet sein, dass das jeweilige Zentrum über entsprechende Möglichkeiten der Kryokonservierung verfügt.

Bei der Kryokonservierung hat sich besonders die Vitrifikation, als extrem schnelles Einfrierverfahren der Oozyten in Stickstoff, bewährt. Bei den konventionellen Methoden erfolgt das Einfrieren deutlich langsamer über mehrere Stunden und birgt ein höheres Risiko für die Bildung von Eiskristallen. Kryokonservierte, fertili- 
sierte Oozyten weisen ähnlich wie kryokonservierte Embryonen oder Spermien eine bei Vitrifikation hohe Vitalität von 73 bis $95 \%$ mit einer Schwangerschaftsrate von etwa $25 \%$ auf [33, 34].

\section{In-vitro-Maturation}

Die In-vitro-Maturation (IVM) ist eine noch recht neue Technik der extrakorporalen assistierten Reproduktionsmedizin, die im Unterschied zur klassischen IVF/ICSI auf eine kontrollierte ovarielle Hyperstimulation verzichtet, sodass es hierbei zu keinem verzögerten Beginn einer geplanten Chemotherapie kommt.

Bei dieser Technik werden, ggf. nach einer kurzen Stimulation mit FSH und/oder hCG, unreife Oozyten aus kleinen antralen Follikeln durch transvaginale Aspiration gewonnen. Wichtig hierbei ist ein hoher antraler Follikelcount (AFC), sodass diese Methode lediglich bei einer kleinen Subgruppe angewandt werden kann.

Es erfolgt dann eine In-vitro-Reifung der Oozyten mit anschließender Fertilisation. Die In-vitro-Maturation der Oozyten wird in aller Regel über 24 Stunden durchgeführt. Anschließend erfolgt eine Beurteilung des Reifestadiums und ggf. die Fertilisation durch konventionelle IVF oder ICSI. Obwohl sich theoretisch die beschleunigte In-vitro-Maturation negativ auswirken könnte, sind bislang keine erhöhten Fehlbildungsraten beschrieben [36]. Nachteile der IVM gegenüber einer konventionellen IVF oder ICSIBehandlung sind [36]:

- geringere Implantations- und Schwangerschaftsraten pro

Therapiezyklus pro Embryotransfer,

- höhere Kosten pro erzielte Schwangerschaft,

- technisch schwierigere Punktion mit längerer Punktionsdauer,

- erhöhte Laborkosten durch erhöhten Arbeitsaufwand,

- nicht vollends geklärte epigenetische und andere fetale Risiken.

Somit handelt es sich bei der In-vitro-Maturation noch um ein experimentelles, nicht standardmäßig angewandtes Verfahren, welches bislang noch in fast keinem Labor angeboten wird.

\section{Kryokonservierung von Ovarialgewebe}

Die Kryokonservierung von Ovarialgewebe hat sich nach ihrer ersten erfolgreichen Anwendung in Brüssel 2004 weltweit als effektives Verfahren etabliert [27]. Hier wurde nach orthotoper, autologer Transplantation zuvor kryokonservierten Ovarialgewebes nach spontaner Konzeption das erste Kind geboren [39].

Bei der Kryokonservierung von Ovarialgewebe werden per Laparoskopie etwa 50 \% des Ovarkortex eines Ovars reseziert, präpariert und unter Verwendung von Kryoprotektiva konserviert. Besonders geeignet ist die Kryokonservierung von Ovarialgewebe für jüngere Patientinnen, da deren Ovarialreserve und somit die Follikeldichte sehr hoch sind. Als obere Altersgrenze wird 35 - 37 Jahre empfohlen, weil bis zu dieser Altersspanne die Follikeldichte des Ovars in den meisten Fällen noch ausreichend hoch ist. Vor der Operation wird der Hormonstatus im Blut bestimmt und eine Sonografie des Uterus und der Ovarien durchgeführt.

Die Kryokonservierung von Ovarialgewebe ist besonders dann geeignet, wenn wenig Zeit bis zum Beginn der zytotoxischen Therapie verbleibt: Während bei der ovariellen Hyperstimulation mit Punktion und Kryokonservierung von Oozyten etwa 2 Wochen eingeplant werden müssen, kann die Laparoskopie wenige Tage vor Beginn der Chemotherapie durchgeführt werden. Der mögliche Verzicht auf eine hormonelle Stimulation, kombiniert mit einem zeitnahen Abschluss der Fertilitätsprotektion, lässt diese Methode, insbesondere im Kollektiv der Mammakarzinompatientinnen, vielversprechend erscheinen.

Bei der Re-Transplantation des Ovarialgewebes wurde in den Anfängen der methodischen Etablierung versucht, das Gewebe heterotop, d. h. im Bereich des Unterarms oder in die Bauchdecke zu transplantieren [40]. Mittlerweile erfolgt die Re-Implantation üblicherweise orthotop, d.h. in oder an das verbliebene Restovar oder in eine Peritonealtasche im Bereich der Fossa ovarica. Innerhalb von 3 bis 6 Monaten kommt es meist zur Wiederaufnahme der endokrinen Funktion. Die Überlebensdauer des Gewebes variiert zwischen wenigen Monaten und mehreren Jahren [41, 42].

Speziell beim Mammakarzinom ist zu beachten, dass ein zumindest theoretisches Restrisiko - für die Re-Induktion der Tumorerkrankung durch persistierende und nach Re-Implantation reaktivierte Mikrometastasen besteht [33]. Gerade in den frühen Stadien (I, II) ist die Re-Transplantation von Ovarialgewebe als sicher anzusehen - sowohl in histologischen als auch immunhistologischen Untersuchungen des kryokonservierten Ovarialgewebes konnten maligne Zellen ausgeschlossen werden. [43, 44]. Im fortgeschrittenen Stadium (IV) hingegen muss das Risiko einer Re-Induktion der Tumorerkrankung durch ovarielle Metastasen zumindest in Betracht gezogen werden. Bei einer Patientin wurde von einem lokalen Rezidiv auf der Seite der erkrankten Brust etwa 1 Jahr nach Re-Transplantation von Ovarialgewebe berichtet. Allerdings konnten in dem Ovar selbst keine Metastasen nachgewiesen werden $[45,46]$. Dieses innovative Verfahren hat sich innerhalb der letzten Jahre immer stärker etabliert und wird mittlerweile auch in Deutschland an ausgewählten Zentren angeboten [27].

Aktuell können anhand publizierter Fälle und mitgeteilter Daten des Netzwerks FertiPROTEKT über 90 Geburten weltweit belegt werden $[47,48]$.

Bei dieser Methode liegt die prozentuale Lebendgeburtenrate bei 28,4 \% je Patientin bei gleichzeitig höheren Schwangerschaftsraten [49]. Van der Ven beschrieb eine Lebendgeburtenrate von $25 \%$ bei einer Schwangerschaftsrate von über $30 \%$ [50].

\section{Medikamentöse Maßnahmen - GnRH-Agonisten}

Die Rationale der Suppression der Keimzellproliferation besteht darin, mit einer zeitlich begrenzten Behandlung mittels $\mathrm{GnRH}$ Analoga einen Zellzyklusarrest zu initiieren, um so die Follikel vor dem gonadotoxischen Einfluss der Chemotherapie zu schützen. Dieses Konzept beruht auf der Beobachtung, dass bei inaktiven Gonaden im präpubertären Alter die Fertilität durch Chemotherapeutika weniger beeinträchtigt wird als in der reproduktiven Lebensphase. Anfangs kommt es bei der Behandlung mit GnRHAgonisten zu einer initialen Freisetzung der Gonadotropine („flare-up“-Effekt), die ungefähr 1 Woche andauert, sodass GnRH-Analoga mindestens 1 Woche vor Beginn der Chemotherapie zum 1. Mal appliziert werden sollten. Die Anwendung erfolgt als Off-Label-Use. 
- Tab. 3 Möglichkeiten des Fertilitätserhalts bei Patientinnen mit Mammakarzinom [59].

\begin{tabular}{|c|c|c|c|c|c|c|c|}
\hline Maßnahme & $\begin{array}{l}\text { experimentelles } \\
\text { vs. Standardver- } \\
\text { fahren }\end{array}$ & $\begin{array}{l}\text { ovarielle } \\
\text { Stimulation } \\
\text { (OS) erfor- } \\
\text { derlich }\end{array}$ & $\begin{array}{l}\text { verzögerter } \\
\text { Beginn der } \\
\text { Chemotherapie }\end{array}$ & $\begin{array}{l}\text { chirurgi- } \\
\text { sche Inter- } \\
\text { vention }\end{array}$ & $\begin{array}{l}\text { Erhalt der } \\
\text { ovariellen } \\
\text { Funktion } \\
\text { (OF) }\end{array}$ & $\begin{array}{l}\text { in allen } \\
\text { Zentren } \\
\text { verfügbar }\end{array}$ & Anmerkungen \\
\hline $\begin{array}{l}\text { Kryokonsvervierung } \\
\text { von unfertilisierten } \\
\text { Oozyten }\end{array}$ & Standard & ja & ja & ja & nein & nein & $\begin{array}{l}\text { Dauer der OS: } \\
\text { 10-14 Tage }\end{array}$ \\
\hline $\begin{array}{l}\text { Kryokonservierung } \\
\text { von fertilisierten } \\
\text { Oozyten }\end{array}$ & Standard & ja & ja & ja & nein & nein & Partner erforderlich \\
\hline In-vitro-Maturation & experimentell & nein & nein & ja & nein & nein & $\begin{array}{l}\text { hoher AFC notwendig, } \\
\text { hohe Kosten, aufwen- } \\
\text { diges Verfahren, wird } \\
\text { bislang in fast keinem } \\
\text { Labor angeboten }\end{array}$ \\
\hline $\begin{array}{l}\text { Kryokonservierung } \\
\text { von Ovarialgewebe }\end{array}$ & experimentell & nein & nein & ja & ja & nein & $\begin{array}{l}\text { nicht zu empfehlen } \\
\text { bei fortgeschrittenem } \\
\text { Tumorstadium, Risiko } \\
\text { Mikrometastasen, kei- } \\
\text { ne Langzeitdaten bez. } \\
\text { der OF }\end{array}$ \\
\hline $\begin{array}{l}\text { ovarielle Suppression } \\
\text { durch GnRH-Analoga }\end{array}$ & experimentell & nein & nein & nein & ja & ja & $\begin{array}{l}\text { einfache, günstige } \\
\text { Therapie, Anwendung } \\
\text { vor und während der } \\
\mathrm{CT} \text {, wenig Langzeit- } \\
\text { daten bez. der Wieder- } \\
\text { aufnahme und dem } \\
\text { Erhalt der OF }\end{array}$ \\
\hline
\end{tabular}

AFC = antraler Follikelcount; $\mathrm{CT}$ = Chemotherapie; GnRH-Analoga = Gonadotropin-releasing Hormon Analoga; OF = ovarielle Funktion; OS = ovarielle Stimulation.

Der Nutzen der GnRH-Analoga wird seit Jahren kontrovers diskutiert. In einer Cochrane-Analyse [52] basierend auf der Auswertung von 4 randomisierten Studien aus den Jahren 1987 bis 2007 zeigten die Autoren, dass die Patientinnen von der Verwendung von GnRH-Analoga profitieren. Es konnten erhöhte Menstruations- (RR 1,90, $95 \%-K I ~ 1,30-2,79)$ und Ovulationsraten (RR 2,70, $95 \%$-KI 1,52 - 4,79) nach abgeschlossener Chemotherapie gezeigt werden [52]. Eine Metaanalyse aus dem Jahr 2011 [53], die 6 Studien bis 2010 einschloss, konnte ebenfalls einen protektiven Effekt durch GnRH-Analoga auf die ovarielle Funktion nachweisen.

Die POEMS (Prevention of Early Menopause Study), eine internationale Phase-III-Studie der Southwest Oncology Group (SWOG), untersuchte den Einfluss von Goserelin auf die Ovarialfunktion während einer Cyclophosphamid-haltigen Chemotherapie. 257 Patientinnen wurden zwischen 2004 und 2011 eingeschlossen und über 2 Jahre nachbeobachtet. Unter Einsatz von GnRH-Analoga konnte eine geringere Rate an prämaturer Ovarialinsuffizienz $(p=0,03)$ im Vergleich zur alleinigen Chemotherapie nachgewiesen werden [54]. Zusätzlich wurden das Disease-free Survival (DFS) sowie das Overall Survival (OS), jeweils bezogen auf 4 Jahre, untersucht, welches sich unter Hinzunahme von Goserelin auf beide Parameter positiv auswirkte (DFS: 89 vs. $78 \%$; OS: 92 vs. $82 \%, p=0,05)[54]$.
Die Daten der POEMS-Studie werden allerdings sehr kritisch bewertet, weil Finanzierungs- und Rekrutierungsprobleme zu einem frühzeitigen Studienabbruch führten. Weiter beeinträchtigt die Interpretation eine zu hohe Drop-out-Rate. Für eine verlässliche Auswertung ist die Studie daher als „unterpowert“ zu werten [55].

Eine Metaanalyse aus dem Jahr 2015 von Lambertini et al. [56] untersuchte 12 randomisierte Studien mit insgesamt 1231 prämenopausalen Mammakarzinompatientinnen. Die temporäre Suppression der Ovarialfunktion durch GnRH-Analoga war mit einem geringeren Risiko eines Preterm ovarian Failure (POF) assoziiert (OR 0,36, 95\%-KI 0,23-0,57; p<0,001) [56].

Bei der PROMISE-GIM6-Studie handelt es sich um eine multizentrische, randomisierte Phase-III-Studie aus dem Jahr 2015 [57]. 281 Patientinnen wurden zwischen 2003 und 2008 eingeschlossen. Die prämenopausalen Patientinnen wurden entweder der Chemotherapiegruppe (Kontrollgruppe) oder der Chemotherapie + GnRH-Analogon (Triptorelin)-Gruppe zugeordnet. Primäre Endpunkte waren ovarielle Funktion, Schwangerschaftsrate und das Disease-free Survival. Menstruationsraten nach Abschluss der Chemotherapie waren mit 72,6\% bei Chemotherapie + Triptorelin bzw. $64 \%$ bei alleiniger Chemotherapie angegeben (HR 1,28, $95 \%-K I$ 0,98-1,68; $\mathrm{p}=0,071)$. Das 5-Jahres-DFS war mit 80,5\% 
bzw. 83,7\% für Chemotherapie + Triptorelin bzw. alleiniger Chemotherapie angegeben (HR 1,17, 95\%-KI 0,72-1,92; p=0,519) [57]. Zusammenfassend kommt die Studie zu der Schlussfolgerung, dass von einem Schutz der Ovarialfunktion durch GnRHAnaloga bei gleichzeitig unveränderter Prognose ausgegangen werden kann.

Zu einem anderen Resultat kamen die Autoren Elgindy und Mitarbeiter 2015. In dieser Arbeit [58] wurden 10, zwischen 1987 und 2015 veröffentlichte, randomisiert kontrollierte Studien identifiziert und ausgewertet. Die Daten von 907 Patientinnen gingen in die Metaanalyse ein, wobei 468 Frauen während der Chemotherapie zusätzlich mit einem GnRH-Agonisten behandelt wurden. Es zeigten sich keine signifikanten Unterschiede zwischen den zusätzlich mit GnRH-Analoga und den allein mittels Chemotherapie behandelten Patientinnen (68,4 vs. 59,9\%). Die Patientinnen profitierten weder hinsichtlich des FSH-Spiegels $(p=0,27)$, des Anti-Müller-Hormons $(p=0,40)$, noch bez. der Antralfollikelzahl $(p=0,17)$ von der GnRH-Analoga-Gabe [58].

Vor dem Hintergrund der widersprüchlichen Aussagen bez. der Wirksamkeit von GnRH-Analoga sollte der Einsatz mit der Patientin kritisch und ausführlich diskutiert werden [5].

- Tab. 3 gibt eine Übersicht über die o. g. Möglichkeiten zum Fertilitätserhalt.

\section{Schlussfolgerung}

1. Alle prämenopausalen Patientinnen mit nicht abgeschlossener Familienplanung sollten vor einer gonadotoxischen Chemotherapie über die Möglichkeiten des Fertilitätserhalts aufgeklärt werden.

2. Je nach Tumorstadium und Tumorbiologie und möglichem Zeitintervall sollten die zur Verfügung stehenden Maßnahmen individuell mit der Patientin besprochen werden.

3. Die verschiedenen fertilitätserhaltenden Maßnahmen können kombiniert werden.

4. Eine ovarielle Hyperstimulation kann unabhängig vom Zyklustag zu jedem Zeitpunkt begonnen werden.

5. Dies gilt für hormonrezeptorpositive wie auch -negative Mammakarzinome gleichermaßen.

6. GnRH-Agonisten werden weiterhin kontrovers diskutiert, sollten aber der Patientin nicht vorenthalten werden.

7. Die laparoskopische Entnahme von Ovarialkortex ist eine vielversprechende Methode, die den Beginn einer Chemotherapie nicht verzögert.

Interessenkonflikt

Die Autoren geben an, dass kein Interessenkonflikt besteht.

\section{Erstveröffentlichung}

Dieser Beitrag wurde erstveröffentlicht in: Geburtshilfe Frauenheilkd 2017; 77(10): 1088 - 1094. DOI: 10.1055/s-0043-119543
Literatur

[1] Allemani C, Weir HK, Carreira H, CONCORD Working Group et al. Global surveillance of cancer survival 1995-2009: analysis of individual data for 25676887 patients from 279 population-based registries in 67 countries (CONCORD-2). Lancet 2015; 385: 977 - 1010

[2] Gesellschaft der epidemiologischen Krebsregister in Deutschland e.V. Atlas der Krebsinzidenz und -mortalität in Deutschland (GEKID-Atlas). 2013. Online: http://www.gekid.de last access: 04.06.2017

[3] Barnes B, Bertz ], Buttmann-Schweiger N et al. Bericht zum Krebsgeschehen in Deutschland 2016. Zentrum für Krebsregisterdaten im Robert Koch Institut, November 2016. Berlin: Robert Koch-Institut. 2016; 22: $34-35$

[4] Robert Koch-Institut. Zentrum für Krebsregisterdaten. Krebs in Deutschland. 3.14 Brustdrüse. 2012. Online: http://www.krebsdaten.de last access: 04.06.2017

[5] Liedtke C, Kiesel L. Chemotherapy-induced amenorrhea - an update. Geburtsh Frauenheilk 2012; 72: 809-818

[6] Ritzinger P, Dudenhausen JW, Holzgreve W. Risiken der späten Mutterschaft. Speculum - Zeitschrift für Gynäkologie und Geburtshilfe 2012; 30: $15-23$

[7] Pronzato P, Mustacchi G, De Matteis A et al. Biological characteristics and medical treatment of breast cancer in young women-a featured population: results from the NORA study. Int J Breast Cancer 2011; 2011: 534256

[8] Alvarez RM, Ramanathan P. Fertility preservation in female oncology patients: the influence of the type of cancer on ovarian stimulation response. Hum Reprod 2016. doi:10.1093/humrep/dew158

[9] Creux H, Monnier P, Son WY et al. Immature oocyte retrieval and in vitro oocyte maturation at different phases of the menstrual cycle in women with cancer who require urgent gonadotoxic treatment. Fertil Steril 2017; 107: 198-204

[10] Ataya KM, McKanna JA, Weintraub AM et al. A luteinizing hormone-releasing hormone agonist for the prevention of chemotherapy-induced ovarian follicular loss in rats. Cancer Res 1985; 45: $3651-3656$

[11] Familiari G, Caggiati A, Nottola SA et al. Ultrastructure of human ovarian primordial follicles after combination chemotherapy for Hodgkin's disease. Hum Reprod 1993; 8: 2080-2087

[12] Dann EJ, Epelbaum R, Avivi I et al. Fertility and ovarian function are preserved in women treated with an intensified regimen of cyclophosphamide, adriamycin, vincristine and prednisone (Mega-CHOP) for nonHodgkin lymphoma. Hum Reprod 2005; 20: 2247-2249

[13] Walshe JM, Denduluri N, Swain SM. Amenorrhea in premenopausal women after adjuvant chemotherapy for breast cancer. J Clin Oncol 2006; 24: 5769-5779

[14] Zhou W, Ding Q, Liang X et al. The risk of amenorrhea is related to chemotherapy-induced leucopenia in breast cancer patients receiving epirubicin and taxane based chemotherapy. PLoS One 2012; 7: e37249

[15] Fornier MN, Modi S, Panageas KS et al. Incidence of chemotherapy-induced, long-term amenorrhea in patients with breast carcinoma age 40 years and younger after adjuvant anthracycline and taxane. Cancer 2005; 104: 1575-1579

[16] Martin M, Pienkowski T, Mackey J, Breast Cancer International Research Group 001 Investigators et al. Adjuvant docetaxel for node-positive breast cancer. N Engl J Med 2005; 352: 2302-2313

[17] Meirow D, Nugent $D$. The effects of radiotherapy and chemotherapy on female reproduction. Hum Reprod Update 2001; 7: 535 - 543

[18] Christinat A, Pagani O. Fertility after breast cancer. Maturitas 2012; 73 : $191-196$

[19] Ruddy KJ, Partridge AH. The unique reproductive concerns of young women with breast cancer. Adv Exp Med Biol 2012; 732: 77-87 
[20] Ruddy KJ, O’Neill A, Miller KD et al. Biomarker prediction of chemotherapy-related amenorrhea in premenopausal women with breast cancer participating in E5103. Breast Cancer Res Treat 2014; 144: 591 - 597

[21] Reimer T, Kempert S, Gerber B et al. SLCO1B1*5 polymorphism (rs4149056) is associated with chemotherapy-induced amenorrhea in premenopausal women with breast cancer: a prospective cohort study. BMC Cancer 2016; 16: 337

[22] Day FR, Ruth KS, Thompson DJ et al. Large-scale genomic analyses link reproductive aging to hypothalamic signaling, breast cancer susceptibility and BRCA1-mediated DNA repair. Nat Genet 2015; 47: 1294-1303

[23] Hickey M, Peate M, Saunders CM et al. Breast cancer in young women and its impact on reproductive function. Hum Reprod Update 2009; 15: $323-339$

[24] Pagani O, Ruggeri M, Manunta S et al. Pregnancy after breast cancer: Are young patients willing to participate in clinical studies? Breast 2015; 24: $201-207$

[25] International Breast Cancer Study Group. Pregnancy outcome and safety of interrupting therapy for women with endocrine responsive breast cancer (POSITIVE) - Trial. NCT 02308085. 2016. Online: https://clinicaltrials.gov/ct2/show/study/NCT02308085 last access: 08.06.2017

[26] Camp-Sorrell D. Cancer and its treatment effect on young breast cancer survivors. Semin Oncol Nurs 2009; 25: 251 - 258

[27] Carter J, Chi DS, Brown CL et al. Cancer-related infertility in survivorship. Int J Gynecol Cancer 2010; 20: 2 - 8

[28] Penrose R, Beatty L, Mattiske J et al. The psychosocial impact of cancerrelated infertility on women: a review and comparison. Clin J Oncol Nurs 2013; 17: 188-193

[29] von Wolff M. Perspektive Fertilität: Indikation und Durchführung fertilitätsprotektiver Maßnahmen bei onkologischen und nicht-onkologischen Erkrankungen. Kiel: Verlag Schmidt \& Klaunig; 2016

[30] von Wolff M, Thaler C], Frambach T et al. Ovarian stimulation to cryopreserve fertilized oocytes in cancer patients can be started in the luteal phase. Fertil Steril 2009; 92: 1360-1365

[31] Oktay K, Buyuk E, Libertella $\mathrm{N}$ et al. Fertility preservation in breast cancer patients: a prospective controlled comparison of ovarian stimulation with tamoxifen and letrozole for embryo cryopreservation. J Clin Oncol 2005; 23: $4347-4353$

[32] Oktay K, Hourvitz A, Sahin G et al. Letrozole reduces estrogen and gonadotropin exposure in women with breast cancer undergoing ovarian stimulation before chemotherapy. J Clin Endocrinol Metab 2006; 91: $3885-3890$

[33] Czeromin UV. Deutsches IVF-Register (DIR). Gablitz, Österreich: Krause \& Pachernegg GmbH, Verlag für Medizin und Wirtschaft; 2015: 24-25

[34] Desai N, Blackmon H, Szeptycki ] et al. Cryoloop vitrification of human day 3 cleavage-stage embryos: post-vitrification development, pregnancy outcomes and live births. Reprod Biomed Online 2007; 14: 208 213

[35] Nawroth F. Kryokonservierung. In: Nawroth F Social Freezing. Wiesbaden: Springer Fachmedien; 2015: 88 - 90

[36] Diedrich K, Ludwig M, Griesinger G. Reproduktionsmedizin. Berlin: Spinger-Verlag; 2013: $248-251$

[37] Kidder BL. In vitro maturation and in vitro fertilization of mouse oocytes and preimplantation embryo culture. Methods Mol Biol 2014; 1150: $191-199$

[38] Benkhalifa M, Demirol A, Ménézo Y et al. Natural cycle IVF and oocyte invitro maturation in polycystic ovary syndrome: a collaborative prospective study. Reprod Biomed Online 2009; 18: 29-36

[39] Donnez J, Dolmans MM, Demylle D et al. Livebirth after orthotopic transplantation of cryopreserved ovarian tissue. Lancet 2004; 364 : $1405-1410$

[40] Shamonki MI, Oktay K. Oocyte and ovarian tissue cryopreservation: indications, techniques, and applications. Semin Reprod Med 2005; 23: 266 - 276
[41] Macklon KT, Jensen AK, Loft A et al. Treatment history and outcome of 24 deliveries worldwide after autotransplantation of cryopreserved ovarian tissue, including two new Danish deliveries years after autotransplantation. J Assist Reprod Genet 2014; 31: 1557 - 1564

[42] Dittrich R, Hackl J, Lotz L et al. Pregnancies and live births after 20 transplantations of cryopreserved ovarian tissue in a single center. Fertil Steril 2015; 103: $462-468$

[43] Donnez J, Dolmans MM, Pellicer A et al. Restoration of ovarian activity and pregnancy after transplantation of cryopreserved ovarian tissue: a review of 60 cases of reimplantation. Fertil Steril 2013; 99: 1503-1513

[44] Dolmans MM, Luyckx V, Donnez J et al. Risk of transferring malignant cells with transplanted frozen-thawed ovarian tissue. Fertil Steril 2013; 99: 1514-1522

[45] Rosendahl M, Greve T, Andersen CY. The safety of transplanting cryopreserved ovarian tissue in cancer patients: a review of the literature. J Assist Reprod Genet 2013; 30: $11-24$

[46] Rosendahl M, Timmermans Wielenga V, Nedergaard L et al. Cryopreservation of ovarian tissue for fertility preservation: no evidence of malignant cell contamination in ovarian tissue from patients with breast cancer. Fertil Steril 2011; 95: 2158-2161

[47] Jensen AK, Macklon KT, Fedder J et al. 86 successful births and 9 ongoing pregnancies worldwide in women transplanted with frozen-thawed ovarian tissue: focus on birth and perinatal outcome in 40 of these children. J Assist Reprod Genet 2017; 34: 325 - 336

[48] FertiPROTEKT N. FertiPROTEKT, Jahrestreffen Innsbruck. 2017. Online: http://www.fertiprotekt.com last access: 04.06.2017

[49] Pacheco F, Oktay K. Current success and efficiency of autologous ovarian transplantation: a meta-analysis. Reprod Sci 2017; 24: 1111 - 1120

[50] Van der Ven H, Liebenthron ], Beckmann M et al. Ninety-five orthotopic transplantations in 74 women of ovarian tissue after cytotoxic treatment in a fertility preservation network: tissue activity, pregnancy and delivery rates. Hum Reprod 2016; 31: 2031 - 2041

[51] Andersen CY. Success and challenges in fertility preservation after ovarian tissue grafting. Lancet 2015; 385: $1947-1948$

[52] Chen H, Li ], Cui T et al. Adjuvant gonadotropin-releasing hormone analogues for the prevention of chemotherapy induced premature ovarian failure in premenopausal women. Cochrane Database Syst Rev. 2011. (11) CD008018

[53] Bedaiwy MA, Abou-Setta AM, Desai N et al. Gonadotropin-releasing hormone analog cotreatment for preservation of ovarian function during gonadotoxic chemotherapy: a systematic review and meta-analysis. Fertil Steril 2011; 95: 906 -914.e1-906-14.e4

[54] Moore HC, Unger JM, Phillips KA, POEMS/S0230 Investigators et al. Goserelin for ovarian protection during breast-cancer adjuvant chemotherapy. N Engl J Med 2015; 372: $923-932$

[55] Gerber B, Ortmann O. Muss der Ovarschutz mit GnRHa nach dem ASCO 2014 neu bewertet werden? Frauenarzt 2015; 56: 142 - 145

[56] Lambertini M, Ceppi M, Poggio F et al. Ovarian suppression using luteinizing hormone-releasing hormone agonists during chemotherapy to preserve ovarian function and fertility of breast cancer patients: a metaanalysis of randomized studies. Ann Oncol 2015; 26: 2408-2419

[57] Lambertini M, Boni L, Michelotti A et al. Ovarian suppression with triptorelin during adjuvant breast cancer chemotherapy and long-term ovarian function, pregnancies, and disease-free survival: a randomized clinical trial. JAMA 2015; 314: 2632 - 2640

[58] Elgindy E, Sibai H, Abdelghani A et al. Protecting ovaries during chemotherapy through gonad suppression: a systematic review and metaanalysis. Obstet Gynecol 2015; 126: 187-195

[59] Tomasi-Cont N, Lambertini M, Hulsbosch S et al. Strategies for fertility preservation in young early breast cancer patients. Breast 2014; 23: 503 - 510 